\title{
An elastic-plastic constitutive model for ceramic composite laminates
}

\author{
Varun P. Rajan*, John H. Shaw, Michael N. Rossol, Frank W. Zok \\ Materials Department, University of California, Santa Barbara, CA 93106, United States
}

\section{A R T I C L E I N F O}

\section{Article history:}

Received 15 April 2014

Received in revised form 19 June 2014

Accepted 21 June 2014

Available online 14 July 2014

\section{Keywords:}

A. Ceramic-matrix composites (CMCs)

B. Mechanical properties

C. Computational modeling

C. Finite element analysis

\begin{abstract}
A B S T R A C T
Existing phenomenological constitutive models are unable to capture the full range of behaviors of ceramic composite laminates. To ameliorate this deficiency, we propose a new model based on the deformation theory of plasticity. The predictive capabilities of the model are assessed through comparisons of computed and measured strain and displacement fields in open-hole tension tests. The agreements in the magnitude of strains and in the size and shape of shear bands that develop around a hole are very good over most of the loading history. Some discrepancies are obtained at high stresses. These are tentatively attributed to non-proportional stressing of some material elements: a feature not captured by the present model.
\end{abstract}

(c) 2014 Elsevier Ltd. All rights reserved.

\section{Introduction}

Ceramic matrix composites (CMCs) of engineering interest exhibit some capacity for inelastic straining prior to fracture. The inelasticity can play an important role in the distributions of stresses and strains in coupons or components containing holes or notches (much like plasticity in metals). One consequence is a reduced degree of notch-sensitivity of tensile strength (relative to truly brittle materials) [1-9]. A robust capability for modeling inelasticity in CMCs would enable the design of complex engineering components and could lead to designs that are less conservative than those that would emerge from purely elastic analyses. The present article focuses on the modeling of the deformation of CMCs under multiaxial (2-D) loadings.

The primary mechanism for inelasticity in CMCs at low stresses is matrix microcracking. In notched panels of CMCs with stiff matrices (e.g. $\mathrm{SiC} / \mathrm{SiC}, \mathrm{SiC} / \mathrm{CAS}$ ), microcracking occurs first directly ahead of the notch tip and then spreads across the net section in a spatially distributed manner $[10,1,5]$. In contrast, microcracking in CMCs with compliant matrices (e.g. $\mathrm{SiC} / \mathrm{C}, \mathrm{C} / \mathrm{C}$ ) is manifested in shear damage bands that initiate at the notch tip and extend parallel to the axis of applied load [10,3]. The former type of composite has been termed 'Class II' and the latter type 'Class III' [11]. For both classes, fiber fracture becomes important at higher stresses, providing an additional mechanism of inelastic deformation and ultimately leading to macroscopic rupture.

\footnotetext{
* Corresponding author. Tel.: +1 (805)893 2694.

E-mail address: varun_rajan@umail.ucsb.edu (V.P. Rajan).
}

Micromechanical models developed over the past three decades have provided great insights into the mechanics of matrix cracking. The onset of matrix cracking in unidirectionally-reinforced materials loaded in uniaxial tension along the fiber direction is particularly well-understood $[12,13]$. But such models are difficult to extend in a generic manner to laminates with other architectures and to the macro-scale. There are two reasons for this. First, principal stresses are generally oriented at an arbitrary angle with respect to the fiber axes; existing micromechanical models of even unidirectional CMCs have yet to be extended to general multiaxial in-plane loadings. Second, the mechanisms of cracking in multidirectional laminates and the associated mechanics are significantly more complex than those of the constituent unidirectional plies. Several additional damage mechanisms can arise, including crack tunneling in transverse plies, growth of partially unbridged cracks into axial plies, and delamination between plies [14,15]. It would appear that deriving macro-scale constitutive models (at scales $\gg 1 \mathrm{~mm}$ ) directly from micromechanical models (at scales of $1 \mu \mathrm{m}$ to $100 \mu \mathrm{m}$ ) is not currently feasible.

Unsurprisingly, existing constitutive models for CMC laminates under general multiaxial loading are largely phenomenological (not micromechanical) in nature [16]. By 'phenomenological', we mean that the models take as inputs macroscopic quantities such as stress-strain curves into a framework that relates stresses to strains. Two classes of models exist.

The first relies on concepts from continuum damage mechanics [17-19]. Here the state of the composite is described by internal damage variables that can be scalars, vectors, or tensors. As these damage variables evolve with applied loads, the stiffness of the composite is degraded. The damage evolution laws are calibrated 
using experimental data. In some models, such as that of Talreja [17], the damage variables are, in principle, measurable quantities, e.g. crack density; in others, such as that of Camus [18], they are not, and must instead be inferred from macroscopic stressstrain data. The fundamental tradeoff in all such models is between the complexity of the representation of damage and the amount of experimental data needed for calibration. For instance, the model of Talreja [17] requires evolution laws for four different damage tensors that represent crack densities, debond lengths, etc. A very extensive experimental program is thus required to calibrate the model for general multiaxial loading. Despite their complexity, it remains unclear whether the predictive capability of such models is actually enhanced by the large number of internal variables.

The second modeling approach dispenses with internal damage variables and instead assumes that the degradation of the composite stiffness can be related to macroscopic stress-strain functions from standard mechanical tests that elicit the important damage mechanisms. In principle, this approach should yield mathematically simpler models that can be calibrated with far fewer experimental data, since there are far fewer 'fitting constants.' Models of this type have been developed mainly for use with polymer matrix composites [20-22]. An attempt to extend the approach to CMC laminates was made by Genin and Hutchinson [2] and later expanded by Rajan and Zok [23]. For reasons elaborated upon in Section 3, however, these models are limited in applicability; that is, they presuppose a particular form of the yield/cracking surface that may be consistent with the behavior of some material systems but not others. Here we address these deficiencies and propose an alternative model that is less restrictive yet equally straightforward to implement.

The principal objective of the present study is to develop a plane-stress, phenomenological elastic-plastic model for CMC laminates that satisfies the following criteria:

1. It can be calibrated using experimental data from standard mechanical tests (e.g. tension, shear).

2. It is applicable to common CMC fiber architectures, notably cross-ply $\left(\left[0^{\circ} / 90^{\circ}\right]_{s}\right)$ and quasi-isotropic $\left(\left[0^{\circ} / \pm 45^{\circ} / 90^{\circ}\right]_{s}\right)$ laminates.

3. It can capture the behavior of a range of CMCs, including those with stiff or compliant matrices.

The outline of the article is as follows. First, the results of mechanical tests (uniaxial tension at $0^{\circ}$, tension at $45^{\circ}$, and Iosipescu shear) for a commercial $\mathrm{SiC} / \mathrm{SiCN}$ woven $\mathrm{CMC}$ are presented. Second, these data are combined with previously-reported results for other material systems and used to assess two existing phenomenological models for CMC laminates: notably, that of Genin and Hutchinson [2] and an adaptation of the model of Hahn [20]. The two models are distinguished by their respective predictions of the relationship between the mechanical responses in shear and in $45^{\circ}$ tension. Notably, the shear/tensile cracking stress ratios from the GH model and the Hahn model are 1 and $1 / 2$, respectively. The actual behavior, from both the experimental data and from theoretical considerations, is roughly bounded by these two extremes. Third, a new phenomenological elastic/plastic model is proposed. It is based on the deformation theory of plasticity and it combines features of both the GH and Hahn-type models. Importantly, it allows for arbitrary values of the shear/tensile cracking stress ratio. The predictive capabilities of the model are assessed in two ways: (i) by comparisons with measured inelastic responses in $45^{\circ}$ tensile stress-strain curves, and (ii) comparisons of computed and measured displacement and strain fields in openhole tension tests. The errors in the predicted open-hole tensile results are computed and rationalized in terms of the degree of non-proportional stressing that occurs during inelastic straining in the test geometry of interest.

\section{Materials and experiments}

\subsection{Material}

The material investigated in the present study comprises 8 plies of $\mathrm{Hi}$-Nicalon ( $\mathrm{SiC}$ ) fibers in an 8-harness satin weave, a BN interface coating, and a SiCN matrix made through a combination of slurry infiltration and precursor impregnation and pyrolysis (S-200H, COI Ceramics, Inc., San Diego, CA). The finished composite panel was $2.25 \mathrm{~mm}$ thick. Optical micrographs (Fig. 1) reveal some remnant porosity and extensive microcracking in the matrix. These defects play a significant role in the average matrix modulus and strength, as manifested in the mechanical test results presented below.

\subsection{Experimental procedures}

Three sets of mechanical tests were performed: uniaxial tension at $0^{\circ}$ and at $45^{\circ}$, and shear parallel to the two fiber directions. Either two or three specimens were used for each set. The test specimens were designed to accommodate the limited quantity of available material.

For both sets of uniaxial tension tests, a dog-bone geometry was employed. For the test at $0^{\circ}$, the gauge width was $12.4 \mathrm{~mm}$ and the gauge length was $25.4 \mathrm{~mm}$; for the test at $45^{\circ}$, the gauge width was $7 \mathrm{~mm}$ and the gauge length was $14 \mathrm{~mm}$. Fiberglass tabs were adhered using a commercial epoxy to the ends of the tensile specimens to promote even load transfer. The specimens were loaded using hydraulic wedge grips.

Shear properties were measured using the Iosipescu test. Specimen design was broadly in accordance with the pertinent ASTM standard (ASTM D5379), with one notable exception: the V-notch angle was selected to be $105^{\circ}$, instead of $90^{\circ}$ (Fig. 2). A notch angle larger than $90^{\circ}$ is desirable for testing orthotropic materials, since it produces somewhat smaller transverse stresses in the central ligament [24]. The specimen was loaded using a standard Iosipescu test fixture (Wyoming Test Fixtures, Inc.).

All specimens were instrumented using strain gauges on one surface and 3-D DIC (VIC-3D, Correlated Solutions, Inc.) on the other. For the Iosipescu shear test, a $\left[0^{\circ} / 90^{\circ}\right]$ stacked strain gauge rosette (Vishay Micro Measurements, CEA-13-062WT-120), oriented at $\pm 45^{\circ}$ to the specimen axes, was used; a single (axial) strain gauge was used for the tension tests. To enable use of digital image correlation, an artificial speckle pattern was created on the sample surface. This was accomplished by first painting the specimen surface uniformly white and then spraying fine black speckles onto the surface using an airbrush. The speckle size, measured using an autocorrelation technique, was approximately $50 \mu \mathrm{m}$. Two digital cameras (Point Grey Research Grasshopper), each with

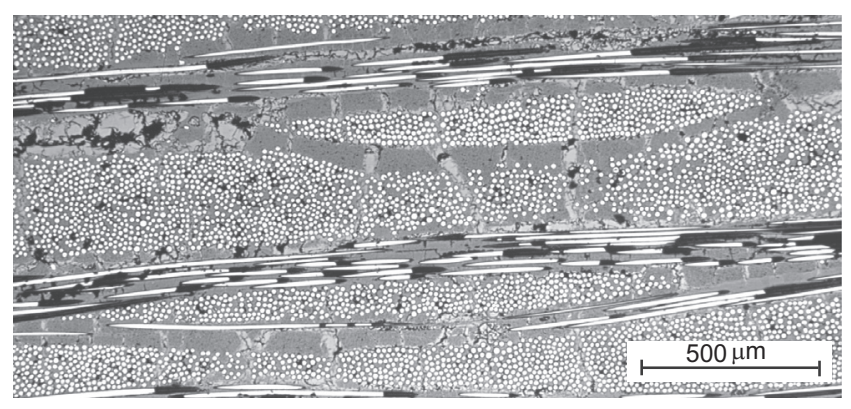

Fig. 1. Optical micrograph of a polished cross-section through the composite. 


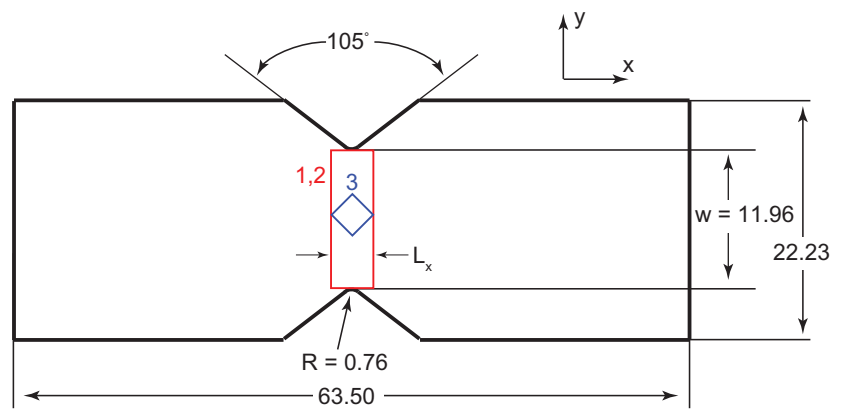

Fig. 2. Schematic of Iosipescu test specimen (dimensions are in millimeters). (For interpretation of the references to color in this figure legend, the reader is referred to the web version of this article.)

a CCD resolution of 2448pixels $\times 2048$ pixels and a $70-180 \mathrm{~mm}$ lens (Nikon ED AF Micro Nikkor), were used to acquire images for DIC. The focal length of the lenses was $70 \mathrm{~mm}$, the aperture setting was $\mathrm{F}-11$, and the angle between cameras ranged between $19^{\circ}$ and $35^{\circ}$. Images were taken with a scale factor of $8 \mu \mathrm{m} /$ pixel to $12 \mu \mathrm{m} /$ pixel. To maximize the spatial resolution of the displacement measurement, the smallest possible subset size that ensured full correlation was chosen. This subset size $\left(h_{\text {sub }}\right)$ was 30 pixels to 45 pixels, or $270-370 \mu \mathrm{m}$. With this choice, the spatial resolution of displacements is well below the in-plane tow dimension (roughly $1 \mathrm{~mm}$ ). The step size was selected to be $h_{\text {sub }} / 10$, rounded to the nearest pixel.

\subsection{Calculation of strains}

Some subtleties are involved in the calculation of strains from the full-field displacement data generated by DIC, particularly for the Iosipescu test. For the tensile tests, the desired quantities are the axial and transverse strains; for the shear test, the desired quantity is the shear strain. These strains must be computed by averaging over a suitably-chosen area, $A$. The straightforward approach is to compute the area-averaged strain, $\bar{\epsilon}$, by differentiating displacements to find local strains and subsequently averaging these quantities over $A$. The average is equivalent to an integral, which is evaluated numerically as follows:

$\bar{\epsilon}_{i j}=\frac{1}{A} \int_{A} \epsilon_{i j}(x, y) \mathrm{d} A \approx \frac{1}{N} \sum_{k \in A} \epsilon_{i j}\left(x_{k}, y_{k}\right) \Delta A_{k}$

where $\left(x_{k}, y_{k}\right)$ are the coordinates of the $k$ th node within $A, \Delta A_{k}$ is its associated area, and $N$ is the number of nodes within $A$. An alternative approach is to utilize the insight of Grédiac et al. [25]: notably, that the integral in Eq. (1) can be transformed using Green's theorem. For instance, for shear strains,

$\bar{\gamma}=\frac{1}{A} \int_{A} \gamma(x, y) \mathrm{d} A=\frac{1}{A} \oint_{\partial A} v(x, y) \mathrm{d} y-u(x, y) \mathrm{d} x$

where the path integral is taken counterclockwise over the boundary of $A$, denoted $\partial A$. Once again, the integral is evaluated by discretizing over $\partial A$; specifically, the nodal displacement data obtained from DIC is interpolated in $2 \mathrm{D}$ and the integral of interest is evaluated by sampling the interpolation function over $\partial A$. The advantages of the latter approach are twofold. First, it does not require the extra step of computing strains from displacements. Second, it avoids using local strains, which tend to be somewhat 'noisy' (since numerical differentiation is an inherently noisy process [25]). Therefore, the present work utilizes the path integral method for computing area-averaged strains.

\footnotetext{
${ }^{1}$ In practice, however, we find that the two methods give very similar results (differences of less than roughly $2 \%$ ) provided that full correlation is maintained.
}

A second issue involves the selection of $A$. In uniaxial tension tests, the strain is nominally uniform and therefore $A$ is chosen to be the entire area of the sample that lies within the field of view. For the Iosipescu shear test, however, there are competing considerations. Ideally, the experimental data would yield a function that relates the average shear stress to the average shear strain. The average stress is taken over the ligament, which is a rectangle lying within the $y z$-plane. However, the average strain is taken over $A$ which lies within the $x y$-plane. The width $L_{x}$ (in the $x$-direction) of $A$ must be large enough to ensure that a sufficient number of data points is used to compute the average, but small enough to ensure that the strain distribution within the averaging area is uniform. Computations (not shown) indicate that a reasonable compromise is afforded by selecting $L_{x}$ to be $0.8 \mathrm{~mm}$, which is roughly equal to the notch radius, $r$.

A further issue that arises in the Iosipescu test is a discrepancy (on the order of tens of percent) between front-face and back-face strains. These differences arise for two reasons [26,27]. First, the specimen may undergo twisting (torsion) about the $x$-axis [26]. (In the present experiments, DIC measurements of the out-of-plane displacement do indeed reveal some amount of twisting.) Second, Saint-Venant effects arise from the loading because the distance between the central ligament and the inner loading points is small. Either effect is sufficient to create a non-uniform distribution of shear strain in the through-thickness direction, $z$. Moreover, while the former effect can be suppressed by appropriately modifying the Iosipescu test fixture, the latter cannot [27]. However, both effects can be eliminated simply by averaging the front and back-face strains [27]. As a check on the fidelity of the resulting shear strain measurements, the measured shear modulus was compared with that predicted from elasticity using the elastic properties measured in the tension tests: the two agreed to within $5 \%$.

\subsection{Results of mechanical testing}

Axial and transverse strain measurements (from DIC) for the tension tests at $0^{\circ}$ and $45^{\circ}$ are shown in Fig. 3(a) and (b). Shear stress-strain curves from the Iosipescu test are shown in Fig. 3(c). ${ }^{2}$ The latter strains were computed by averaging the DIC and strain gauge data on the two surfaces. The stress-strain curves exhibit little variation between specimens. For the purpose of calibrating the constitutive models (Sections 3 and 4), average stress-strain curves were calculated using a smoothing spline through the experimental data. The averages were computed only in the hardening regime (i.e. post-peak strain-softening was neglected). These curves are also depicted in Fig. 3.

Five elastic constants can be calculated from the five stressstrain curves: the Young's moduli $E_{0}$ and $E_{45}$ in the $0^{\circ}$ and $45^{\circ}$ directions, the corresponding Poisson's ratios $v_{0}$ and $v_{45}$, and the shear modulus $G$. Their average values are summarized in Table 1. From the theory of elasticity, only three of the constants are independent. Treating $E_{0}, v_{0}$, and $G$ as independent, the values of the other two quantities, $E_{45}$ and $v_{45}$, can be expressed as:

$$
\begin{aligned}
E_{45} & =\frac{4 E_{0} G}{E_{0}+2 G\left(1-v_{0}\right)} \\
v_{45} & =\frac{2 E_{0}}{E_{0}+2 G\left(1-v_{0}\right)}-1
\end{aligned}
$$

The predicted values of $E_{45}$ and $v_{45}$ are also shown in Table 1 . The error between the elasticity prediction and the measurement for $E_{45}$ is $3 \%$; the corresponding error for $v_{45}$ is $12 \%$, indicating that the experimental data is reasonably self-consistent.

\footnotetext{
${ }^{2}$ All shear strains referred to herein are engineering shear strains.
} 

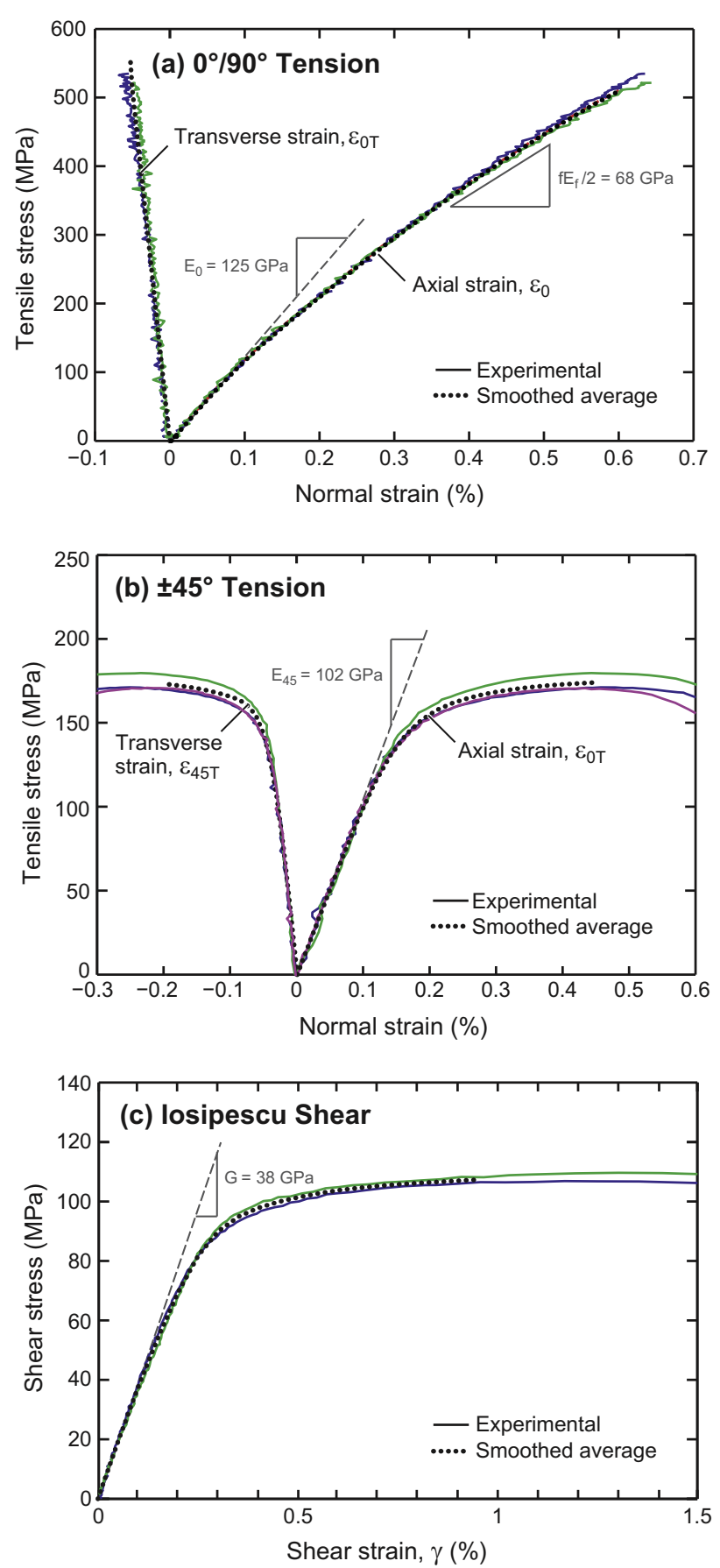

Fig. 3. Measured stress-strain curves for (a) tension at $0^{\circ}$, (b) tension at $45^{\circ}$, and (c) shear. The normal strains in (a) and (b) were computed using DIC data whereas the shear strains in (c) were obtained by averaging the DIC and strain gauge data on the two faces, as described in the text. Also depicted are smoothed averages. (For interpretation of the references to color in this figure legend, the reader is referred to the web version of this article.)

Table 1

Measured elastic constants. Predicted values of $E_{45}$ and $v_{45}$ (from elasticity theory) are also shown.

\begin{tabular}{llllll}
\hline & $E_{0}(\mathrm{GPa})$ & $v_{0}$ & $G(\mathrm{GPa})$ & $E_{45}(\mathrm{GPa})$ & $v_{45}$ \\
\hline Measured & 125 & 0.12 & 38 & 102 & 0.27 \\
Predicted & - & - & - & 99 & 0.30 \\
\hline
\end{tabular}

The $0^{\circ}$ tensile curve exhibits a nearly-bilinear response, with the change in tangent modulus occurring over the range of
$100-200 \mathrm{MPa}$. The change in tangent modulus is relatively small: only about a factor of two. This behavior is reminiscent of that observed in both $\mathrm{C} / \mathrm{C}$ and oxide-oxide composites with porous and/or microcracked matrices. In contrast, the curves in $45^{\circ}$ tension and in shear exhibit nearly elastic-perfectly plastic behavior. In the latter cases, the tangent moduli in the post-cracking regimes are about an order of magnitude smaller than the corresponding elastic moduli. Additionally, the magnitude of the inelastic transverse strains for $45^{\circ}$ loading are comparable to the inelastic axial strains, which is characteristic of a fiber 'scissoring' mechanism [2]. Similar macroscopic stress-strain behavior for off-axis loading has been observed in glass- and carbon-matrix composites [28,29].

The effective elastic modulus of the (porous, microcracked) matrix can be extracted by employing an elastic analysis based on laminate theory (see the appendix for pertinent formulae). Each woven cloth, comprising $0^{\circ}$ and $90^{\circ}$ fibers, is partitioned into two unidirectional plies: one at $0^{\circ}$ and the other at $90^{\circ}$. The volume fraction of fibers within each ply is taken to be equal to that of the overall composite, which is calculated from the manufacturer's reported weave properties (fiber areal weight and fiber density) and the number of plies and thickness of the composite panel. The resulting estimate is $V_{f}=0.52$. The elastic properties of each ply are calculated using a well-accepted micromechanical model: the generalized self-consistent scheme (GSCS) [30,31]. The GSCS model requires the constituent properties. The Young's modulus of the (isotropic) fiber $\left(E_{f}\right)$ is taken from manufacturer data. The porous, cracked matrix is homogenized and represented by an isotropic effective medium with properties $\left(E_{m}\right.$ and $\left.v_{m}\right)$ that are unknown a priori. Using the elastic constants of the plies, the elastic constants of the laminate are readily computed using standard relations from laminate theory. The remaining constituent properties $\left(E_{m}, v_{m}\right.$, and $\left.v_{f}\right)$ are subsequently solved for by minimizing the error between the predicted and experimentally measured elastic constants of the laminate. The resulting property values are: $E_{m}=43 \mathrm{GPa}, v_{m}=0.15$, and $v_{f}=0.18$. Using these values, the agreement between the predicted and measured elastic constants is excellent: the errors being less than $1 \%$.

Taken together, these results imply that, although the matrix in fully-dense form would be very stiff, its effective modulus is significantly less than that of the fibers $\left(E_{m} / E_{f} \approx 0.16\right)$. Similar results have been reported for a 3-D woven $\mathrm{C} / \mathrm{SiC}$ composite [32], in which the SiC matrix was processed using similar methods (precursor impregnation and pyrolysis (PIP) and slurry infiltration). In this material, the effective matrix modulus was roughly $15 \mathrm{GPa}$ : an order of magnitude less than the modulus of dense SiC. The PIP process tends to produce matrices that are highly porous and microcracked, as evidenced by Fig. 1 . The principal reason is that the pre-ceramic polymer undergoes a large volume change upon pyrolysis, which gives rise to void formation and shrinkage cracking. Such pores and cracks may be impossible to re-infiltrate during subsequent PIP cycles because infiltration pathways may be closed off during the initial cycle. Thermal expansion mismatch between the fibers and the matrix (as in the composite studied in Yang et al. [32] may lead to further microcracking. Moreover, small interstices (e.g. between fibers within a tow) may be difficult to infiltrate even in the initial cycle if a high-viscosity, particleloaded slurry is used. All of these factors cause PIP-derived matrices to exhibit high levels of matrix damage (pores, cracks). Therefore, as noted by Flores et al. [33], the effective modulus of such matrices cannot be estimated a priori, as in polymer matrix composites. Instead, the modulus must be calibrated using experimental results.

Because the matrix is very compliant relative to the fibers, the composite behavior is therefore expected to most closely resemble that of 'Class III' composites. For instance, shear banding should be 
the primary near-notch damage mechanism. (That this is indeed the case is demonstrated in Section 5.)

\section{Assessment of existing constitutive models}

\subsection{Preliminaries}

Based on the phenomenological constitutive models described in the introduction, the five stress-strain curves presented above are not all independent. For instance, the $45^{\circ}$ tensile response is predicted to be dependent on the $0^{\circ}$ tensile and shear responses. Here we assess the internal consistency of the in-plane stressstrain curves stemming from both the GH model for CMC laminates [2,23] and a model adapted from the work of Hahn [20] on PMC laminates. The assessment is made using the test data for $\mathrm{SiC} / \mathrm{SiCN}$ described above as well as comparable data previously reported for two other CMCs: (i) a cross-ply SiC/CAS laminate [28], and (ii) a 2-D woven $\mathrm{SiC} / \mathrm{SiC}$ composite [34,18]. The results presented below demonstrate that neither model is able to accurately predict the $45^{\circ}$ tensile stress-strain curve from the $0^{\circ}$ tensile and shear stress-strain curves for all material systems. But, the measured $45^{\circ}$ tensile stress-strain curves are roughly bounded by the predictions of the two models.

In the following analysis, the axial and transverse strains for $0^{\circ}$ tensile loading are represented by the functions $\epsilon_{0}=f_{0}(\sigma)$ and $\epsilon_{0 T}=f_{0 T}(\sigma)$, respectively. Similarly, the axial and transverse strains for $45^{\circ}$ tensile loading are $\epsilon_{45}=f_{45}(\sigma)$ and $\epsilon_{45 T}=f_{45 T}(\sigma)$, respectively. Finally, the shear stress-strain curve is $\gamma=f_{s}(\sigma)$.

\subsection{GH model}

The GH model is couched in terms of a tangent compliance matrix relating principal strains to principal stresses. In the inelastic regime, for principal strains oriented at $45^{\circ}$ to the fibers, the constitutive equation takes the form:

$$
\left[\begin{array}{c}
\epsilon_{I} \\
\epsilon_{I I}
\end{array}\right]=\left[\begin{array}{cc}
f_{45}^{\prime}\left(\sigma_{I}\right) & f_{45 T}^{\prime}\left(\sigma_{I}\right) D_{45}+f_{45 T}^{\prime}\left(\sigma_{I I}\right)\left(1-D_{45}\right) \\
f_{45 T}^{\prime}\left(\sigma_{I}\right) & f_{45}^{\prime}\left(\sigma_{I}\right) D_{45}+f_{45}^{\prime}\left(\sigma_{I I}\right)\left(1-D_{45}\right)
\end{array}\right]\left[\begin{array}{c}
\sigma_{I} \\
\sigma_{I I}
\end{array}\right]
$$

where the primes denote differentiation with respect to stress; the subscripts I, II denote the first and second principal strains/stresses, respectively; and the parameter $D_{45}$ characterizes the tendency of the laminate to 'scissor' when loaded in tension in the $45^{\circ}$ direction. For cross-ply laminates, a reasonable estimate for $D_{45}$ is unity [23]. Eq. (4) can be used to relate the $45^{\circ}$ tensile response to the shear response of the CMC. The result is [23]:

$f_{s}^{\prime}(\sigma) \approx\left(1+D_{45}\right)\left(f_{45}^{\prime}(\sigma)-f_{45 T}^{\prime}(\sigma)\right)$

Eq. (5) implies that if the stress, $\sigma$, is sufficient to cause significant inelasticity for $45^{\circ}$ loading (i.e. to cause $f_{45}^{\prime}(\sigma)$ and $f_{45 T}^{\prime}(\sigma)$ to become large in magnitude), it is sufficient to cause significant inelasticity in shear loading, and vice versa. In other words, the stress-strain curves for $45^{\circ}$ tension and shear loading are expected to exhibit significant nonlinearity at the same stress level.

\subsection{Hahn model}

The Hahn model was originally developed for PMC laminates. It uses the contracted notation for stresses and strains: e.g. $\sigma_{1}=\sigma_{11}, \sigma_{6}=\sigma_{12}, \epsilon_{6}=2 \epsilon_{12}$, etc., where the 1 - and 2-directions are aligned with the fiber axes. The governing equations for crossply laminates in the original formulation of the model are [20]:

$$
\left[\begin{array}{l}
\epsilon_{1} \\
\epsilon_{2} \\
\epsilon_{6}
\end{array}\right]=\left[\begin{array}{ccc}
a_{11} & a_{12} & 0 \\
a_{12} & a_{22} & 0 \\
0 & 0 & a_{66}+S_{6666} \sigma_{6}^{2}
\end{array}\right]\left[\begin{array}{c}
\sigma_{1} \\
\sigma_{2} \\
\sigma_{6}
\end{array}\right]
$$

Here nonlinearity is incorporated only in the shear response (through the quadratic term) and not in the $0^{\circ}$ tensile response [20]. However, the model can be generalized to include nonlinearity in tension by using a tangent compliance formulation:

$$
\left[\begin{array}{l}
\mathrm{d} \epsilon_{2} \\
\mathrm{~d} \epsilon_{2} \\
\mathrm{~d} \epsilon_{6}
\end{array}\right]=\left[\begin{array}{ccc}
f_{0}^{\prime}\left(\sigma_{1}\right) & f_{0 T}^{\prime}\left(\sigma_{2}\right) & 0 \\
f_{0 T}^{\prime}\left(\sigma_{1}\right) & f_{0}^{\prime}\left(\sigma_{2}\right) & 0 \\
0 & 0 & f_{s}^{\prime}\left(\sigma_{6}\right)
\end{array}\right]\left[\begin{array}{l}
\mathrm{d} \sigma_{1} \\
\mathrm{~d} \sigma_{2} \\
\mathrm{~d} \sigma_{6}
\end{array}\right]
$$

(If $f_{0}^{\prime}(\sigma)=a_{11}=a_{22}, f_{0 T}^{\prime}(\sigma)=a_{12}$, and $f_{s}^{\prime}(\sigma)=a_{66}+3 S_{6666} \sigma^{2}$, Eq. (7) reduces to Eq. (6).)

Here, again, the constitutive model can be used to relate the shear and $45^{\circ}$ tensile responses. Doing so yields the relationships:

$$
\begin{aligned}
& f_{45}^{\prime}(\sigma)+f_{45 T}^{\prime}(\sigma)=f_{0}^{\prime}\left(\frac{\sigma}{2}\right)+f_{0 T}^{\prime}\left(\frac{\sigma}{2}\right) \\
& f_{45}^{\prime}(\sigma)-f_{45 T}^{\prime}(\sigma)=\frac{1}{2} f_{s}^{\prime}\left(\frac{\sigma}{2}\right)
\end{aligned}
$$

The latter is similar in form to Eq. (5) in the GH model. However, in contrast to the GH model, the Hahn model predicts that if the $45^{\circ}$ response exhibits nonlinearity at $\sigma$, then the shear response should exhibit nonlinearity at $\sigma / 2$. That is, the predicted cracking stresses for $45^{\circ}$ tension from the $\mathrm{GH}$ and the Hahn models differ by a factor of two.

\subsection{Interpretation of experimental data}

The predicted $45^{\circ}$ tensile stress-strain curves from the two models are shown in Fig. 4 for the three CMCs of interest: $\mathrm{SiC} / \mathrm{SiCN}$ (present work), SiC/CAS [28], and SiC/SiC [18].

For the composites with relatively stiff matrices (Fig. 4(b) and (c)), the GH model yields reasonably accurate predictions of the cracking stresses and the inelastic hardening rates in $45^{\circ}$ tension. The Hahn model, unsurprisingly, performs poorly in these cases, since its prediction of the plateau stress is approximately twice that of the GH model. In contrast, for the composite with a relatively compliant matrix ( $\mathrm{SiC} / \mathrm{SiCN}$, Fig. 4(a)), the Hahn model performs better than the $\mathrm{GH}$ model, though it overestimates the measured stresses by about 25\%. Closer examination of the functional forms of the two models reveals the origin of these differences: the GH model tacitly assumes that inelasticity is governed by the largest principal stress [23] whereas the Hahn model assumes that it is governed by the larger of the tensile and shear stresses oriented with respect to fiber axes.

Experimental data compiled by Cady et al. [28] for several CMCs are broadly consistent with the trends reported here. That is, the ratio of the cracking stress in $45^{\circ}$ tension and shear loading ranges from about 0.6 for stiff matrices (characterized by $E_{m} / E_{f} \gtrsim 1$ ) to roughly 2 for compliant matrices $\left(E_{m} / E_{f} \ll 1\right)$. Furthermore, a theoretical model for steady state matrix cracking in unidirectional CMCs under off-axis loading yields similar results [35]. Specifically, in the absence of residual stress, the predicted ratio of cracking stresses for $45^{\circ}$ tension and shear loading ranges from 1 for a rigid matrix to 2 for an infinitely compliant matrix. The key conclusion is that an alternative (more generalized) formulation is required to accurately capture the in-plane 'yield' surfaces of CMCs.

\section{Formulation of new model}

\subsection{Preliminaries}

The proposed model is based on the deformation theory of plasticity. Here strains are related to stresses via an effective compliance matrix that is a function of an 'effective stress', $\bar{\sigma}$. The effective stress, in turn, is a function of the components of the stress tensor. (In the deformation theory of plasticity for isotropic 

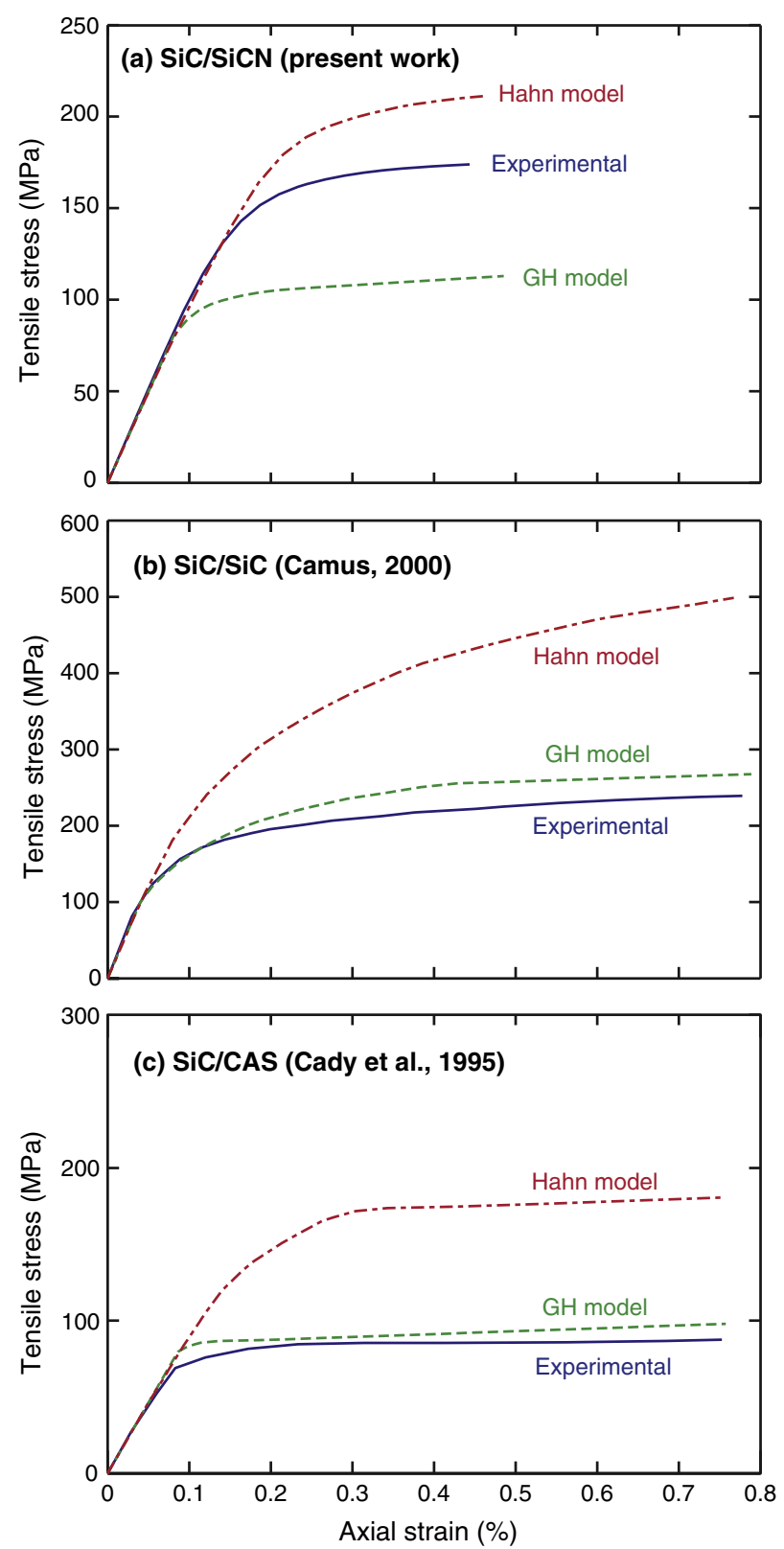

Fig. 4. Comparisons of the $\mathrm{GH}$ and Hahn model predictions of the $45^{\circ}$ axial stressstrain curve with experimental measurements on (a) $\mathrm{SiC} / \mathrm{SiCN}$ composite (from the present work); (b) SiC/SiC composite [18] and (c) SiC/CAS composite [28]). (For interpretation of the references to color in this figure legend, the reader is referred to the web version of this article.)

metals, this would be the von Mises stress.) The effective stress function characterizes the yield/cracking surface of the material. By constructing this function appropriately, the ratio of the cracking stresses for different directions of loading is no longer a constant, as in the GH and Hahn models. Instead, it becomes a fitting parameter that can be calibrated with experimental data. Being a deformation theory, the model is expected to apply for (roughly) proportional stressing. The constitutive relationship is:

$\epsilon_{i}=S_{i j}(\bar{\sigma}) \sigma_{j}$

where $i, j=1,2,6$ (using, again, the contracted notation for stresses and strains). It is assumed that $\bar{\sigma}$ is scale-invariant (a homogeneous function of degree 1 ), which means that:

$\bar{\sigma}\left(C \sigma_{1}, C \sigma_{2}, C \sigma_{6}\right)=C \bar{\sigma}\left(\sigma_{1}, \sigma_{2}, \sigma_{6}\right)$
The cubic in-plane symmetry of the material implies that the 1- and 2-axes are equivalent. Imposing this condition yields the relations:

$S_{11}(\bar{\sigma})=S_{22}(\bar{\sigma})$

$S_{12}(\bar{\sigma})=S_{21}(\bar{\sigma})$

$S_{16}(\bar{\sigma})=S_{26}(\bar{\sigma})$

$S_{61}(\bar{\sigma})=S_{62}(\bar{\sigma})$

$\bar{\sigma}\left(\sigma_{1}, \sigma_{2}, \sigma_{6}\right)=\bar{\sigma}\left(\sigma_{2}, \sigma_{1}, \sigma_{6}\right)$

The functions $S_{i j}(\bar{\sigma})$ are calibrated using the results of only two mechanical tests: notably, $0^{\circ}$ tension and shear aligned with the two fiber axes (the 1- and 2-axes).

\subsection{Mechanical tests for calibration}

\subsubsection{Uniaxial tension at $0^{\circ}$}

For $0^{\circ}$ tension, the effective stress becomes

$\bar{\sigma}=\bar{\sigma}\left(\sigma_{1}, 0,0\right)=C_{0} \sigma_{1}$

using the scale-invariance of $\bar{\sigma}$ (where $C_{0}=\bar{\sigma}(1,0,0)$ ). Eq. (9) then reduces to:

$\epsilon_{1}=S_{11}\left(C_{0} \sigma_{1}\right) \sigma_{1}$

$\epsilon_{2}=S_{21}\left(C_{0} \sigma_{1}\right) \sigma_{1}$

$\epsilon_{6}=S_{61}\left(C_{0} \sigma_{1}\right) \sigma_{1}=0$

The normal strains are given simply by the functions $f_{0}$ and $f_{0 T}$, which characterize the $0^{\circ}$ tensile stress-strain curves. After some algebra, we obtain:

$S_{11}(\bar{\sigma})=S_{22}(\bar{\sigma})=\frac{f_{0}\left(\bar{\sigma} / C_{0}\right)}{\bar{\sigma} / C_{0}}$

$S_{21}(\bar{\sigma})=S_{12}(\bar{\sigma})=\frac{f_{0 T}\left(\bar{\sigma} / C_{0}\right)}{\bar{\sigma} / C_{0}}$

$S_{61}(\bar{\sigma})=S_{62}(\bar{\sigma})=0$

4.2.2. Shear

In pure shear, the effective stress becomes

$\bar{\sigma}=\bar{\sigma}(0,0, \sigma)=C_{s} \sigma$

where $C_{s}=\bar{\sigma}(0,0,1)$. Using a procedure analogous to that utilized above, we obtain:

$S_{66}(\bar{\sigma})=\frac{f_{s}\left(\bar{\sigma} / C_{s}\right)}{\bar{\sigma} / C_{s}}$

$S_{16}(\bar{\sigma})=S_{26}(\bar{\sigma})=\frac{f_{n s}\left(\bar{\sigma} / C_{s}\right)}{\bar{\sigma} / C_{s}}$

where $f_{n s}(\sigma)$ characterizes the normal strain induced by shear stressing. This function is difficult to measure in practice, largely because the strains are exceedingly small in CMCs. Here we assume that $f_{n s}=0$, implying that the shear-extension coupling is negligible.

\subsection{Tangent compliance}

As demonstrated in Section 4.2, the tension and shear tests enable calibration of all of the unknown functions $S_{i j}(\bar{\sigma})$ in the constitutive equation. The final result is:

$$
\left[\begin{array}{c}
\epsilon_{1} \\
\epsilon_{2} \\
\epsilon_{6}
\end{array}\right]=\left[\begin{array}{ccc}
\frac{f_{0}\left(\bar{\sigma} / C_{0}\right)}{\bar{\sigma} / C_{0}} & \frac{f_{0 \mathrm{~T}}\left(\bar{\sigma} / C_{0}\right)}{\bar{\sigma} / C_{0}} & 0 \\
\frac{f_{0 T}\left(\bar{\sigma} / C_{0}\right)}{\bar{\sigma} / C_{0}} & \frac{f_{0}\left(\bar{\sigma} / C_{0}\right)}{\bar{\sigma} / C_{0}} & 0 \\
0 & 0 & \frac{f_{s}\left(\bar{\sigma} / C_{s}\right)}{\bar{\sigma} / C_{s}}
\end{array}\right]\left[\begin{array}{l}
\sigma_{1} \\
\sigma_{2} \\
\sigma_{6}
\end{array}\right]
$$

For ease of numerical implementation, the preceding results are re-expressed in terms of the tangent compliance tensor, $\partial \epsilon_{i} / \partial \sigma_{j}$. Assuming that the stresses are imposed proportionally, i.e. $\sigma_{i}=\Lambda_{i} \bar{\sigma}$, and differentiating Eq. (17), we obtain: 
$\left[\begin{array}{l}\mathrm{d} \epsilon_{1} \\ \mathrm{~d} \epsilon_{2} \\ \mathrm{~d} \epsilon_{6}\end{array}\right]=\left[\begin{array}{ccc}f_{0}^{\prime}\left(\bar{\sigma} / C_{0}\right) & f_{0 T}^{\prime}\left(\bar{\sigma} / C_{0}\right) & 0 \\ f_{0 T}^{\prime}\left(\bar{\sigma} / C_{0}\right) & f_{0}^{\prime}\left(\bar{\sigma} / C_{0}\right) & 0 \\ 0 & 0 & f_{s}^{\prime}\left(\bar{\sigma} / C_{s}\right)\end{array}\right]\left[\begin{array}{c}\mathrm{d} \sigma_{1} \\ \mathrm{~d} \sigma_{2} \\ \mathrm{~d} \sigma_{6}\end{array}\right]$

where the prime denotes differentiation with respect to stress. Eqs. (17) and (18) are mathematically equivalent for proportional stressing; the magnitude of the differences that arise when the stressing is non-proportional is presently unknown.

It is often useful to understand the conditions under which the constitutive model predicts the development of material instabilities, such as tensile or shear localizations [23]. (Ideally, the model should capture the existence of physically realistic instabilities.) These conditions are readily obtained from the tangent compliance matrix (Eq. (18)): material instability arises if the eigenvalues of this matrix are negative. By inspection, we see that the conditions for instability are $f_{s}^{\prime}\left(\bar{\sigma} / C_{s}\right)<0$ or $f_{0}^{\prime}\left(\bar{\sigma} / C_{0}\right)+f_{0 T}^{\prime}\left(\bar{\sigma} / C_{0}\right)<0$. These quantities on the left-hand-sides of these inequalities are physically equivalent to the shear and biaxial compliances, respectively.

\subsection{Predicted response for $45^{\circ}$ tension}

For $45^{\circ}$ tension, the effective stress becomes

$\bar{\sigma}=\bar{\sigma}\left(\frac{\sigma_{45}}{2}, \frac{\sigma_{45}}{2}, \frac{\sigma_{45}}{2}\right)=C_{45} \sigma_{45}$

where $C_{45}=\bar{\sigma}(1 / 2,1 / 2,1 / 2)$. Employing the constitutive equation (Eq. (18)), we obtain:

$\mathrm{d} \epsilon_{1}=\mathrm{d} \epsilon_{2}=\frac{1}{2}\left[f_{0}^{\prime}\left(\frac{C_{45}}{C_{0}} \sigma_{45}\right)+f_{0 T}^{\prime}\left(\frac{C_{45}}{C_{0}} \sigma_{45}\right)\right] \mathrm{d} \sigma_{45}$

$\mathrm{d} \epsilon_{6}=\frac{1}{2} f_{s}^{\prime}\left(\frac{C_{45}}{C_{s}} \sigma_{45}\right) \mathrm{d} \sigma_{45}$

Rewriting these equations in terms of $f_{45}$ and $f_{45 T}$ yields:

$f_{45}^{\prime}\left(\sigma_{45}\right)+f_{45 T}^{\prime}\left(\sigma_{45}\right)=f_{0}^{\prime}\left(\frac{C_{45}}{C_{0}} \sigma_{45}\right)+f_{0 T}^{\prime}\left(\frac{C_{45}}{C_{0}} \sigma_{45}\right)$

and

$f_{45}^{\prime}\left(\sigma_{45}\right)-f_{45 T}^{\prime}\left(\sigma_{45}\right)=\frac{1}{2} f_{s}^{\prime}\left(\frac{C_{45}}{C_{s}} \sigma_{45}\right)$

Eqs. (21) and (22) imply that the difference between axial and transverse strains at $45^{\circ}$ is related to the shear response, whereas their sum is related to the $0^{\circ}$ tensile response. It is instructive to compare Eq. (22) with the corresponding equations for the GH and Hahn models (Eqs. (5) and (8), respectively). When $C_{s}=C_{45}$, the $\mathrm{GH}$ model result is recovered (with $D_{45}=1$ ). When $C_{s}=2 C_{45}$, the Hahn model result is recovered. In general, the model can capture an arbitrary ratio of cracking stresses in $45^{\circ}$ tension and shear.

\subsection{Effective stress}

Heretofore we have derived general results using an arbitrary function $\bar{\sigma}=\bar{\sigma}\left(\sigma_{1}, \sigma_{2}, \sigma_{6}\right)$. For numerical implementation of the constitutive model, a specific function for the effective stress is required. To this end, we use a Hill-type effective stress (which straightforwardly extends the von Mises effective stress to anisotropic materials):

$\bar{\sigma}=\sqrt{A_{i j} \sigma_{i} \sigma_{j}}$

After using material symmetry, this reduces to

$\bar{\sigma}=\sqrt{A_{11}\left(\sigma_{1}^{2}+\sigma_{2}^{2}\right)+2 A_{12} \sigma_{1} \sigma_{2}+A_{66} \sigma_{6}^{2}}$

One limitation of the effective stress in its current form is that it yields equivalent results in compression and in tension. To prevent spurious inelasticity in compression, we replace $\sigma_{1}$ and $\sigma_{2}$ with $R\left(\sigma_{1}\right)$ and $R\left(\sigma_{2}\right)$, where $R$ is the ramp function $(R(x)=(|x|+x) / 2)$.
Finally, the constitutive model requires the constants $C_{0}, C_{45}$ and $C_{s}$. Using the definition of the effective stress, we find:

$$
\begin{aligned}
& C_{0}=\sqrt{A_{11}} \\
& C_{s}=\sqrt{A_{66}} \\
& C_{45}=\frac{1}{2} \sqrt{2 A_{11}+2 A_{12}+A_{66}}
\end{aligned}
$$

Since the Hill-type effective stress essentially defines a yield surface, the constants $A$ (or, equivalently, the constants $C$ ) must be related to the stresses at which the various stress-strain curves exhibit significant non-linearity. Taking these stresses to be $\sigma_{0}, \tau_{0}$ and $\sigma_{45}$, for the three respective tests, we find that

$C_{0} \sigma_{0}=C_{s} \tau_{0}=C_{45} \sigma_{45}$

Using this equation, the constants $A$ in the effective stress function can be evaluated (up to an arbitrary scale factor).

\subsection{Comparison to experimental results}

The present model predicts the $45^{\circ}$ tensile response from the $0^{\circ}$ tension and shear responses. (The prediction is not completely independent, since $\sigma_{45}$ must be specified in order to solve for the unknown constants in the effective stress function. The plastic strains beyond cracking, however, are predicted independently of the experimental measurements.) Results are shown in Fig. 5 for the three material systems examined in Fig. 4. The stresses at which the stress-strain curves start to exhibit significant non-linearity are found by inspection. For the $\mathrm{SiC} / \mathrm{SiCN}$ composite, $\sigma_{0}=170 \mathrm{MPa}$, $\tau_{0}=82.5 \mathrm{MPa}$, and $\sigma_{45}=135 \mathrm{MPa}$; for the SiC/CAS composite [28], $\sigma_{0}=60 \mathrm{MPa}, \tau_{0}=75 \mathrm{MPa}$, and $\sigma_{45}=70 \mathrm{MPa}$; and for the $\mathrm{SiC} / \mathrm{SiC}$ composite [18], $\sigma_{0}=\sigma_{45}=145 \mathrm{MPa}$ and $\tau_{0}=170 \mathrm{MPa}$.

The comparisons show that the agreement between measured and predicted stress-strain curves is excellent. The agreement supports the relationships presented in Eq. (22): notably, that the strain-hardening rates in the shear and $45^{\circ}$ tensile stress-strain curves are related by the scaling factor $C_{s} / C_{45}$. The $\mathrm{GH}$ model assumes this factor to be 1 , while the Hahn-type model assumes it to be 2 . The experimental data indicates that this ratio varies across materials: increasing with decreasing matrix stiffness. The present constitutive model appears to have enough generality to adequately capture this behavior.

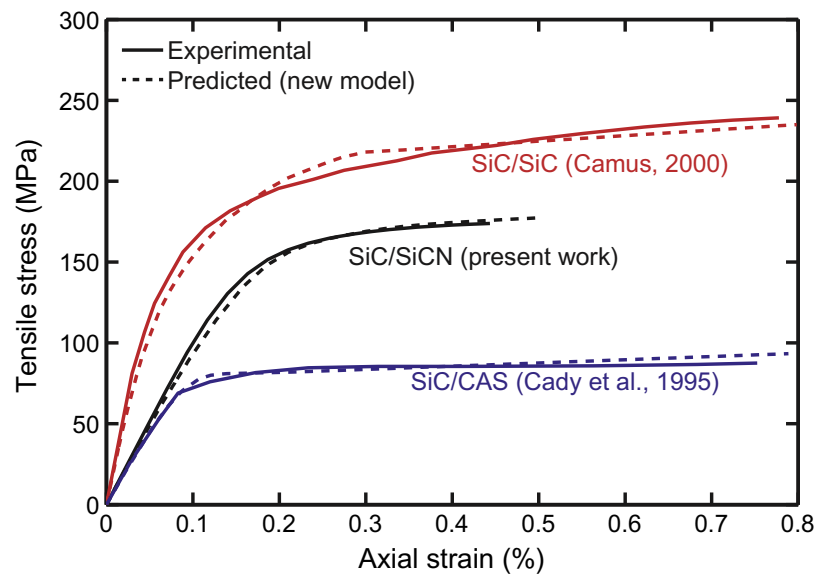

Fig. 5. Comparisons of predicted and measured $45^{\circ}$ axial stress-strain curves for the three composites in Fig. 4. (For interpretation of the references to color in this figure legend, the reader is referred to the web version of this article.) 


\section{Case study: open hole tension}

The constitutive model developed in Section 4 is assessed through comparisons of predicted and measured displacements and strains in open-hole tension tests. Since all calibration data for the model were obtained entirely from (unnotched) tension and shear tests, the comparisons provide a true assessment of the predictive capability of the model.

\subsection{Experiments}

Rectangular specimens of length $114.3 \mathrm{~mm}$ and width $25.4 \mathrm{~mm}$ were used. Holes of $9.525 \mathrm{~mm}$ diameter (37.5\% of the total width) were machined using a diamond core drill. Two specimens were tested: one to failure, and the other to roughly $90 \%$ of the failure stress. As in the unnotched tension tests, fiberglass tabs were adhered to the specimen ends and the specimens were loaded using hydraulic wedge grips.

Digital image correlation was again used to track specimen deformation. To maximize spatial resolution, only about one half of the full specimen width in the vicinity of the hole was imaged.

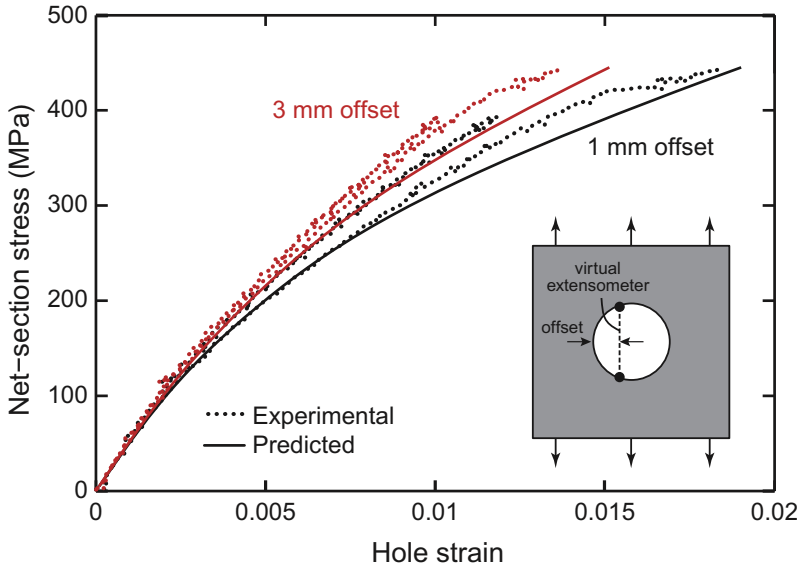

Fig. 6. Comparisons of measured and predicted stress-strain curves for the openhole tension test. The strain is that of a virtual extensometer that spans the hole and is offset from the hole edge, as shown in the inset. Strains were computed for two offsets: $1 \mathrm{~mm}$ and $3 \mathrm{~mm}$. The hole radius is $4.76 \mathrm{~mm}$. (For interpretation of the references to color in this figure legend, the reader is referred to the web version of this article.)

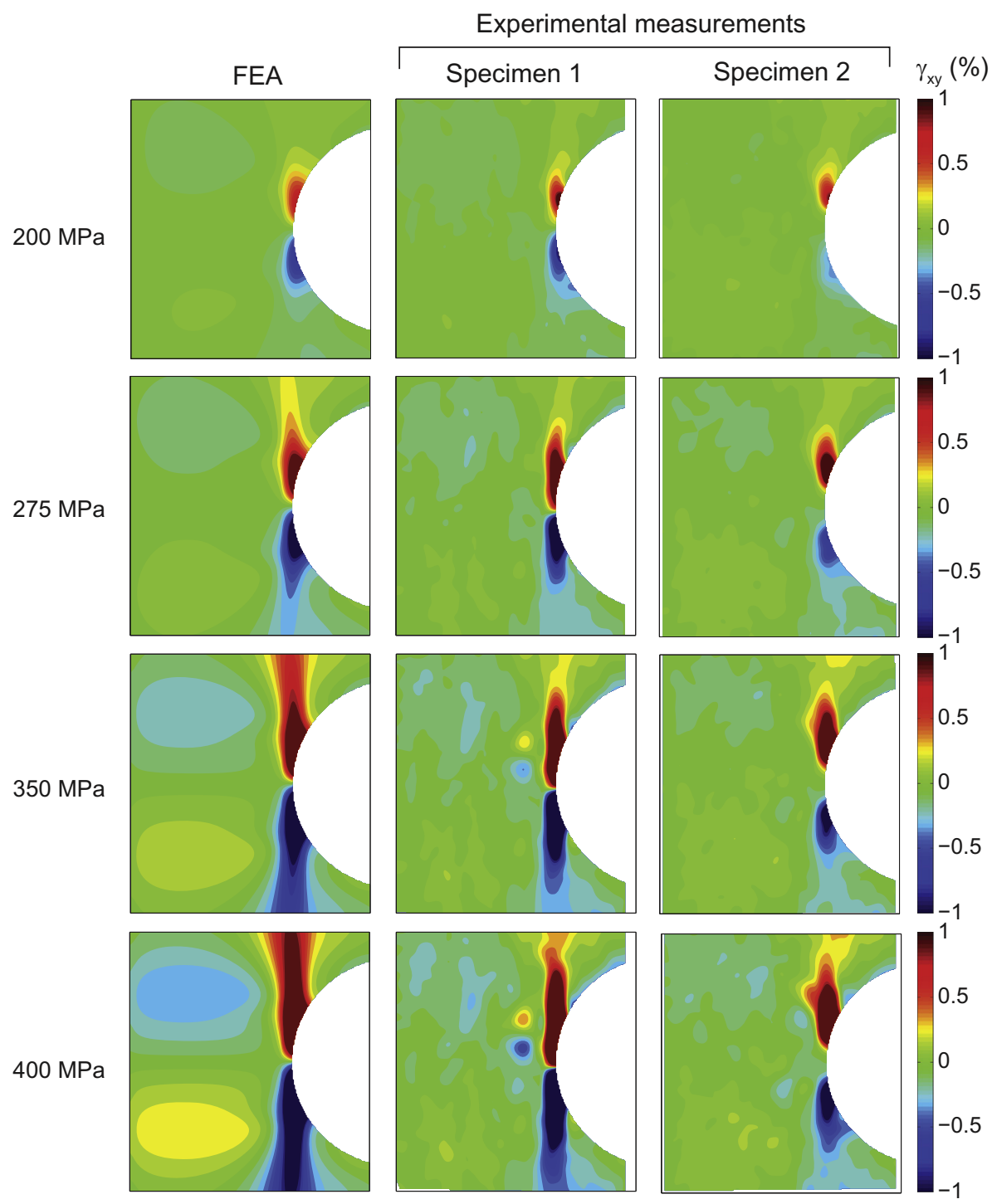

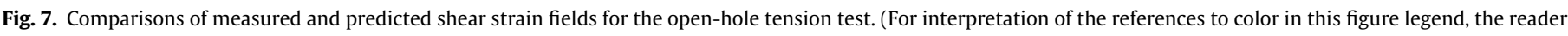
is referred to the web version of this article.) 


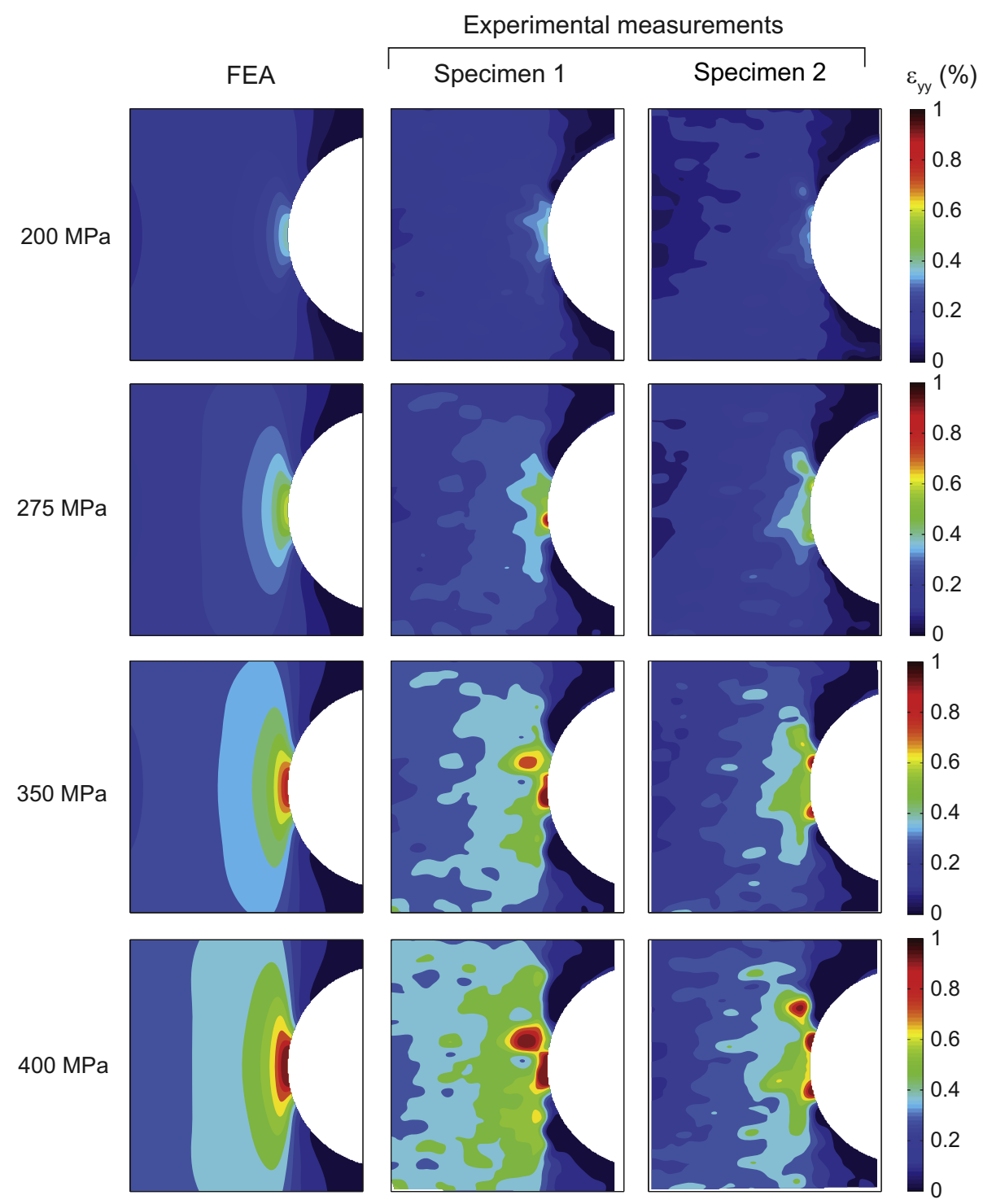

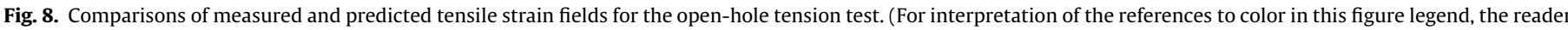
is referred to the web version of this article.)

Parameters for DIC were similar to the tension and shear tests, with the exception of the scale factor, which was slightly smaller (5.5-6 $\mu \mathrm{m} /$ pixel).

\subsection{Finite element simulations}

The constitutive model (Eq. (18)) was implemented in a usermaterial subroutine (UMAT) for use in ABAQUS. The equations were integrated explicitly using the modified Euler scheme described by [36]. The scheme utilizes automatic sub-stepping to limit the error arising from the integration procedure to within a prescribed error tolerance for each time step.

The subroutine utilizes the measured functions $f_{0}, f_{0 T}$, and $f_{s}$ and the constants $C$. The smoothed, averaged stress-strain functions depicted in Fig. 3 are used for this purpose. (Note that these curves neglect material softening.) The constants $C$ are identical to those used previously (found by substituting $\sigma_{0}=170 \mathrm{MPa}, \tau_{0}=$ $82.5 \mathrm{MPa}$, and $\sigma_{45}=135 \mathrm{MPa}$ into Eq. (26)).

The finite element (FE) simulation was performed in ABAQUS Standard (Version 6.12-1, Dassault Systèmes). A quarter-symmetry FE model was employed, with four-noded, quadrilateral, plane-stress elements. The hole diameter was 0.375 of the plate width, as in the experiments. Displacement was monotonically applied at the top boundary in the $y$-direction. A study was performed to ensure that the quantities of interest (nodal displacements) converged with respect to mesh density. For comparison with the experimental data, the displacements $(u, v)$ of every node within the model were extracted at 100 evenly spaced time increments, where the final time increment corresponds to an applied stress which exceeds the failure stress in the experiments.

\subsection{Metrics for comparison}

Several metrics were employed for comparison of experimental results with the FE simulations. To assess the agreement in global response, the nodal displacement data from experiments and simulation were used to compute macroscopic stress-strain curves. In this case, the net-section stress was plotted against the 'hole strain,' measured using a virtual longitudinal extensometer that spans the hole and is offset from the hole edge (see inset of Fig. 6). To assess the correlations in the local fields, contour plots of the displacement in the direction of loading $(v)$ and of the axial 
and shear strains ( $\epsilon_{y y}$ and $\gamma_{x y}$, respectively) were constructed. The (scattered) data from DIC and from FEA were interpolated in $x, y$ using Delaunay triangulation and subsequently evaluated on a common grid in $x-y$ space. Strains were computed from displacements using an identical procedure for both sets of data. Specifically, the gridded data were differentiated with respect to the spatial coordinate using forward differences. The resulting strains were averaged using a Gaussian filter. The filter length, $h_{f}$, is physically equivalent to the gauge length for strain computation. It was selected to be equal to the tow width $(1 \mathrm{~mm})$, since the strains used to calibrate the constitutive model are macroscopic, tow-averaged strains. The standard deviation of the filter was $0.25 h_{f}$.

\subsection{Results}

Macroscopic stress-strain curves from the FE simulation and from the two test specimens are shown in Fig. 6. Two values of the offset, $1 \mathrm{~mm}$ and $3 \mathrm{~mm}$, were selected. (The latter corresponds to the largest offset that lies within the field of view of the cameras.) At low stresses ( $<50 \mathrm{MPa})$, the macroscopic stress-strain behavior is linear and the moduli from the simulation and the measurements are in excellent agreement. At higher stresses (50-300 MPa), the specimens exhibit significant macroscopic nonlinearity. The discrepancies between predicted and measured strains remain small, and less than the sample-to-sample variation. At yet higher stress levels ( $>300 \mathrm{MPa}$ ), the agreement deteriorates somewhat: the simulated strains being slightly larger than the measured values (by roughly 10-30\%). (The fracture stress cannot be predicted since the constitutive law lacks an appropriate failure criterion.)

The shear strain distributions are depicted in Fig. 7. With increasing stress, bands of large shear strain (in excess of 1\%) develop near the edge of the hole and expand parallel to loading direction. The shear bands are a consequence of the low shear cracking stress. The FE simulations appear to predict the size and shape of these bands with good accuracy. At yet higher stress levels (> $400 \mathrm{MPa}$ ), discrepancies between experiment and simulation become progressively more pronounced. However, as discussed later, these stresses fall outside the regime of proportional stressing. Some differences are also obtained between test specimens and between the two shear bands within an individual specimen. (The FE simulation assumes these bands to be identical, since the deformation is symmetric.) We attribute these effects to variations in fiber placement, matrix porosity, and other microstructural characteristics.

Predicted and experimental tensile strain fields are shown in Fig. 8; corresponding line scans of tensile strain along the net-section are plotted in Fig. 9(a). Here the measured strains do not vary smoothly in space. We surmise that there are multiple effects at play. First, there is measurement error associated with digital image correlation. For typical values of displacement noise and the filter length employed in the strain calculation $\left(h_{f}=1 \mathrm{~mm}\right)$, the strain error in regions of relatively uniform strain should be on the order of a few hundred microstrain [37]. This value is evidently insufficient to explain all of the strain inhomogeneity. Second, there are stochastic variations in microstructural characteristics, as mentioned previously. Finally, there are also non-stochastic effects associated with the woven fiber architecture. [37] observed that, in 2-D woven composites, local strain enhancement occurs at tow 'crossovers': locations where undulating warp tows dive beneath flat weft tows. Similar effects may be responsible for the strain 'hotspots' observed in Fig. 8. Despite some noisiness of the measured strain fields, however, the magnitude of the peak strains and their spatial decay away from the hole are similar to those seen in the simulated strain fields. The differences become more pronounced at stresses exceeding about

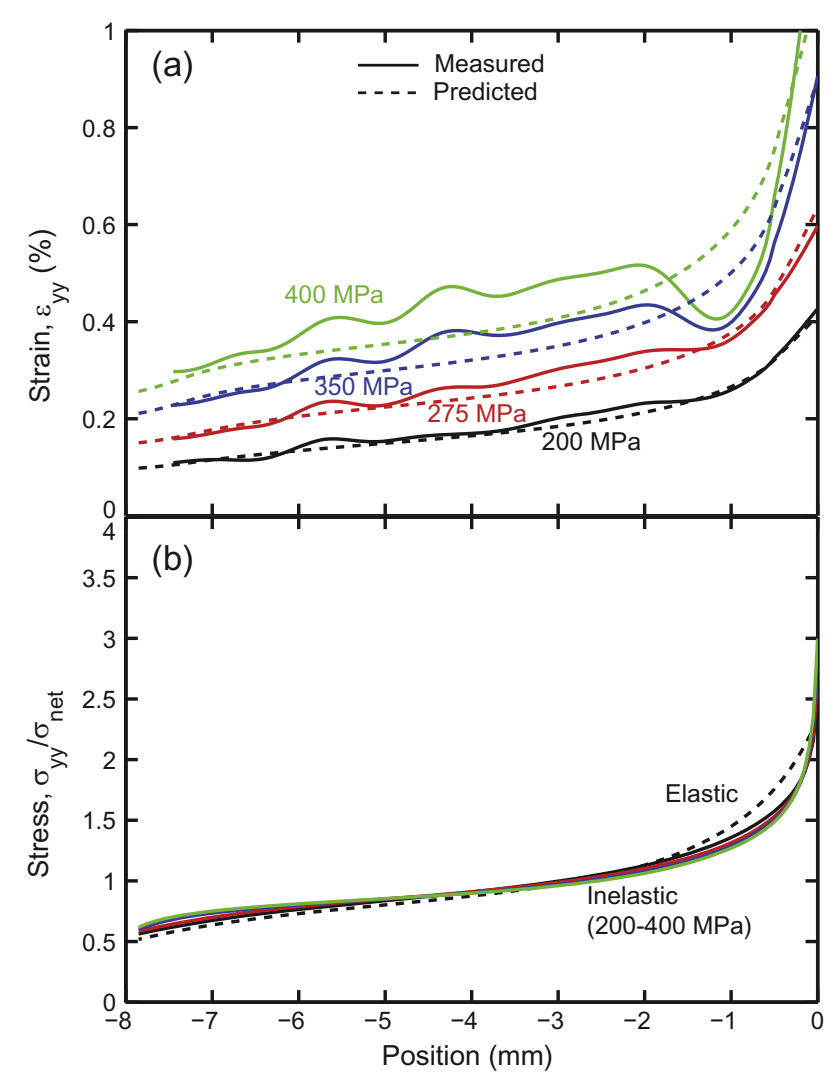

Fig. 9. (a) Measured and predicted strains and (b) predicted stresses along the netsection plane for the open-hole tension test. (For interpretation of the references to color in this figure legend, the reader is referred to the web version of this article.)

$350 \mathrm{MPa}$. In this domain, the tensile strains exceed the (unnotched) tensile failure strain over a length scale comparable to the tow width $(1 \mathrm{~mm})$. We expect, therefore, that significant fiber failure has occurred at this point in the experiment. At even higher stresses, a crack initiates at the hole edge and propagates across the ligament, leading to ultimate failure. Tensile softening due to fiber fracture and concomitant localization of deformation into a crack cannot be captured with the current modeling approach. Approaches based on cohesive zone concepts would be more suitable for this task (see, for instance, He et al. [38], Suo et al. [39].

The agreement between the measured and simulated axial displacements, $v$, shown in Fig. 10, is similarly good, particularly for smaller values of applied stress. (The correlation is unsurprising, since both $\gamma_{x y}$ and $\epsilon_{y y}$ are related to $v$.) The accuracy of these predictions is assessed on the basis of the difference between predicted and experimental displacements, each normalized by the 'hole displacement'-the displacement (from the FE simulation) of a longitudinal extensometer that spans the hole diameter. The results are also plotted in Fig. 10 for one of the test specimens. At stresses below about $200 \mathrm{MPa}$, the errors are very low ( $<5 \%$ everywhere). The errors increase at higher stresses, especially within the shear bands and (to a lesser extent) in the hole 'wake' (the regions above and below the hole, to the right of the shear bands).

Finally, we utilize the simulation results to investigate nearnotch stresses. (These quantities cannot be measured experimentally.) Fig. 9(b) depicts local tensile stresses along the net-section, normalized by the applied net-section stress. In contrast to the local strain fields, no gauge averaging is performed to compute the stresses. As seen, inelasticity does not reduce the stress concentration factor directly at the hole edge, but it does reduce stresses over distances of 1-2 mm from the hole edge. Interestingly, these results are broadly consistent with the 'point stress' fracture 


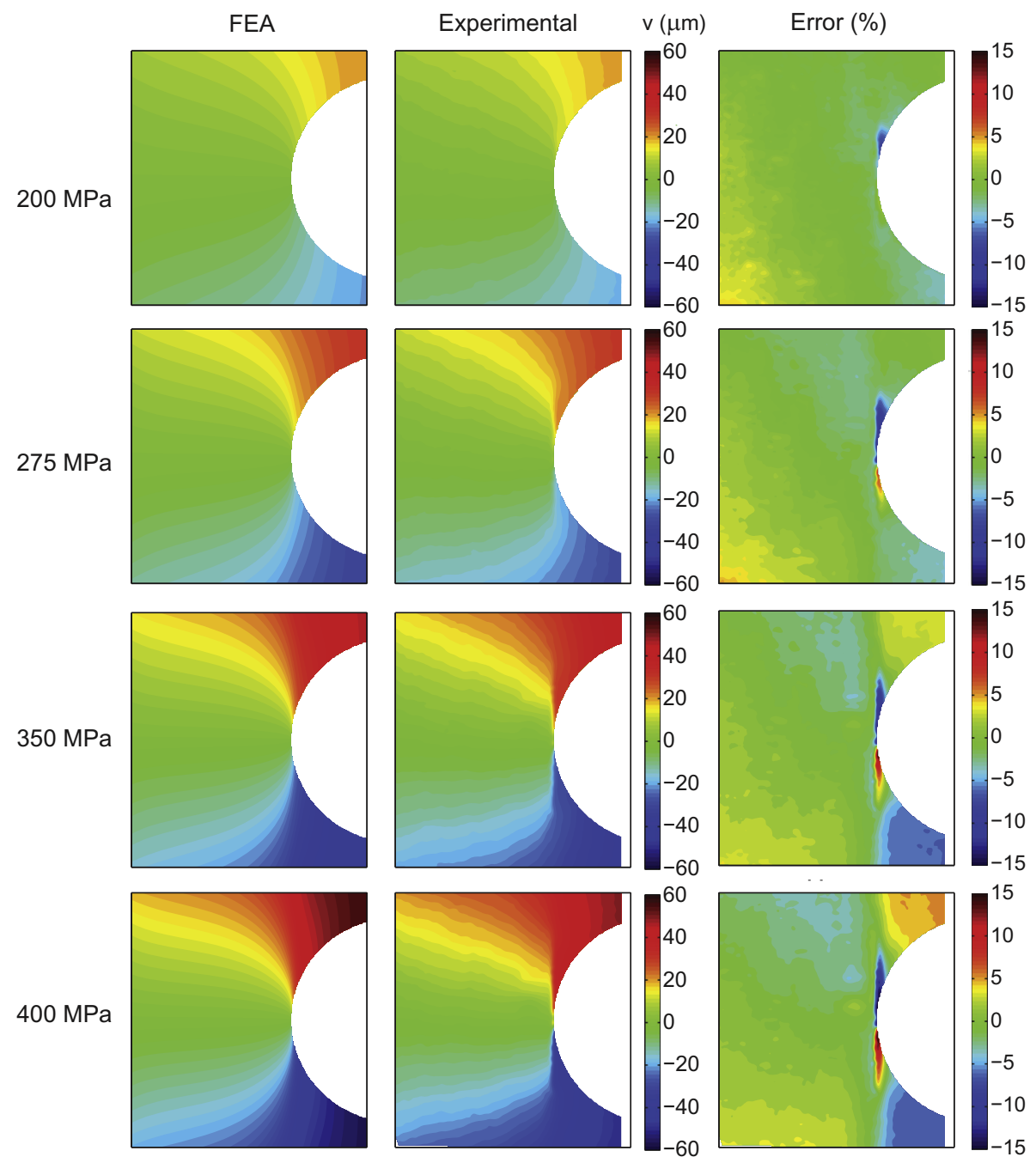

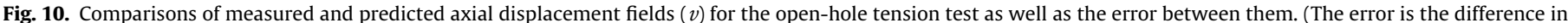

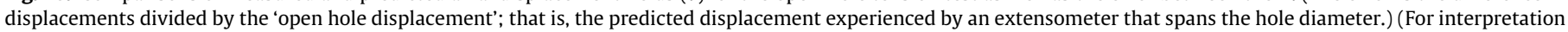
of the references to color in this figure legend, the reader is referred to the web version of this article.)

criterion [40], which is based on the attainment of a critical stress over a characteristic length. It has previously been used to rationalize the notch sensitivity of PMCs [41] and CMCs [7]. In those studies, it had been found that, regardless of the matrix material, the characteristic length is on the order of $1 \mathrm{~mm}$. The data presented herein is broadly consistent with these results; indeed, taking the critical stress to be the unnotched tensile strength, the inferred characteristic length is $1.3 \mathrm{~mm}$.

\subsection{Discussion}

The agreement between predicted and experimental local displacement and strain fields mirrors the agreement between predicted and experimental global extensometer strains. Specifically, the agreement is excellent for smaller values of net-section stress (less than roughly $300 \mathrm{MPa}$ ), and noticeable discrepancies arise only at higher stresses. We speculate that these discrepancies arise because, at higher stresses, the stress state within some material elements follows a non-proportional trajectory. The constitutive model, on the other hand, being based on deformation theory, assumes proportional stressing within every material element.

Deviations from proportional stressing can be assessed by computing ratios using the effective stress:

$$
\begin{aligned}
& \lambda_{x x}=\frac{\bar{\sigma}\left(\sigma_{x x}, 0,0\right)}{\bar{\sigma}\left(\sigma_{x x}, \sigma_{y y}, \sigma_{x y}\right)}=\frac{\sigma_{x x} \sqrt{A_{11}}}{\bar{\sigma}} \\
& \lambda_{y y}=\frac{\bar{\sigma}\left(0, \sigma_{y y}, 0\right)}{\bar{\sigma}\left(\sigma_{x x}, \sigma_{y y}, \sigma_{x y}\right)}=\frac{\sigma_{y y} \sqrt{A_{11}}}{\bar{\sigma}} \\
& \lambda_{x y}=\frac{\bar{\sigma}\left(0,0, \sigma_{x y}\right)}{\bar{\sigma}\left(\sigma_{x x}, \sigma_{y y}, \sigma_{x y}\right)}=\frac{\sigma_{x y} \sqrt{A_{66}}}{\bar{\sigma}}
\end{aligned}
$$

These ratios are of order unity. For instance, if the state of stress within the element is pure tension in the $y$-direction, then $\lambda_{y y}=1$. If, instead, it is pure shear, $\lambda_{y y}=0$. Therefore, changes in $\lambda$ on the order of a few tenths over the loading history indicate significant non-proportional stressing. (Note, however, that only two of the ratios are independent.) Changes in $\lambda$ are of consequence only if they are in regions of significant plastic strains. (In the elastic regime, non-proportional stressing does not induce errors.)

The absolute values of changes in $\lambda_{x y}$ and $\lambda_{y y}$ (with respect to their values in the regime of macroscopic elasticity) are depicted in Fig. 11 for two values of net-section stress. There are two locations where significant changes in $\lambda$ occur: 1 ) in the hole wake and 2) at the shear bands. In the former locations, the stresses are small and the plastic strains are negligible. However, in the latter location, plastic strains are obviously substantial. We speculate 


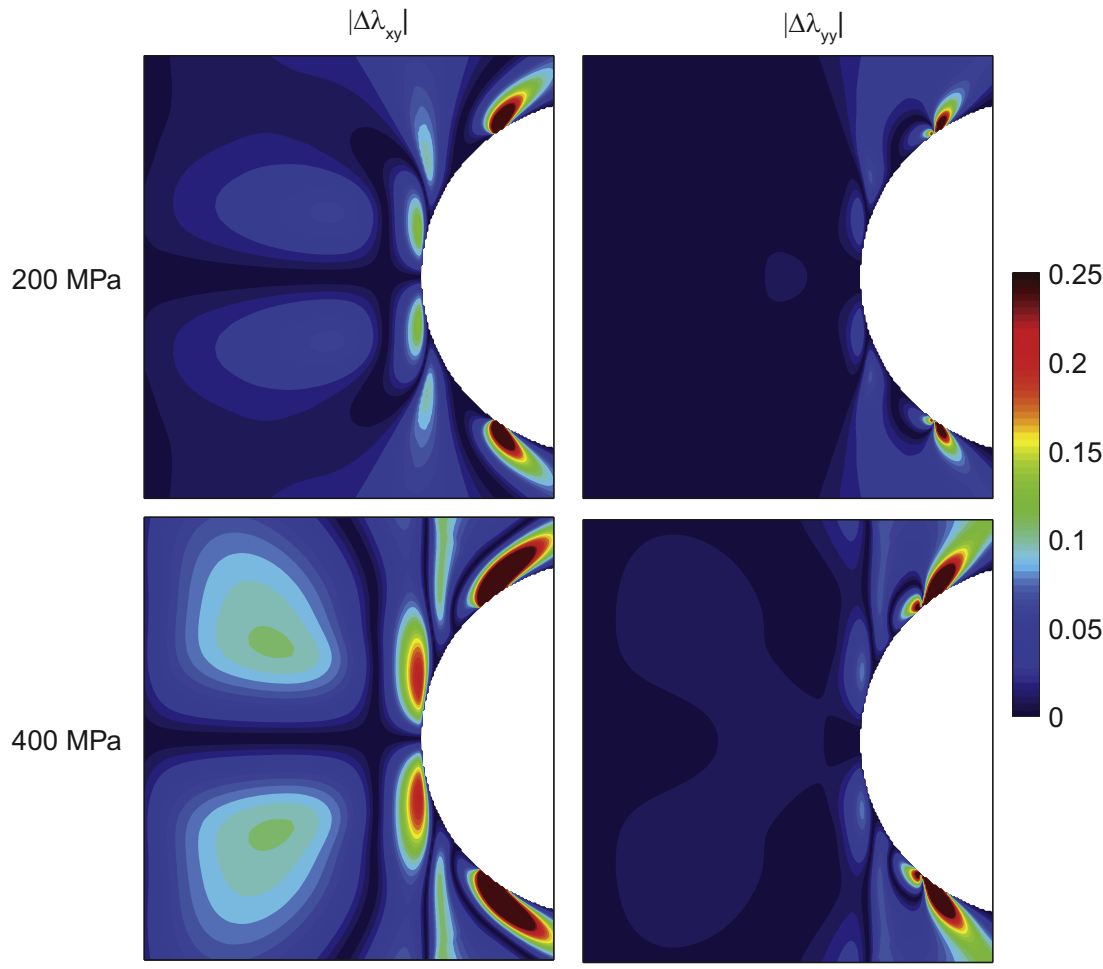

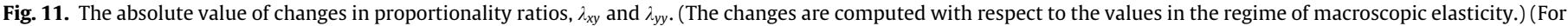
interpretation of the references to color in this figure legend, the reader is referred to the web version of this article.)

that non-proportional stressing within the shear bands is related to the errors in displacement fields observed in Fig. 10. If this hypothesis is correct, then tracking the ratios $\lambda$ over the course of a simulation would provide some insight (albeit qualitative) into the regimes in which the constitutive model is expected to yield accurate results.

It should also be noted that the stress-strain curves used to calibrate the constitutive model omit any description of material softening. Errors arise from this assumption when the tensile and shear strains exceed the values corresponding to the peak tensile and shear stresses (roughly $0.63 \%$ and $1.0 \%$, respectively). In the simulation, the condition for shear softening $\left(\gamma>\gamma_{\text {peak }}\right)$ is first met at a net-section stress of approximately $220 \mathrm{MPa}$. Softening is expected to occur over a significant length scale (the tow width, $1 \mathrm{~mm}$ ) at approximately $270 \mathrm{MPa}$. The corresponding values for tensile softening are $260 \mathrm{MPa}$ and $290 \mathrm{MPa}$, respectively. These results imply that the effects of softening may become relevant when net-section stresses exceed roughly 250-300 MPa. Note, however, that material softening does not explain the discrepancies between measured and simulated displacement/strain fields: incorporation of softening would increase the compliance of the simulated specimen, causing the correlation between prediction and experiment to worsen.

\section{Summary and concluding remarks}

Existing elastic-plastic constitutive models for CMC laminates are insufficiently general; each presupposes a specific form of the yield/cracking surface that is unable to predict the responses of CMCs with both stiff and compliant matrices. In particular, the GH model assumes the ratio of cracking stresses in shear and $45^{\circ}$ tension to be 1 ; the Hahn-type model assumes it to be $1 / 2$. Through a careful examination of experimental data (from mechanical tests reported in the present work and from the literature), we have demonstrated that, in general, CMCs exhibit behaviors that lie roughly between these two extremes. Armed with this insight, a new elastic-plastic model, based on the deformation theory of plasticity, has been developed. It permits an arbitrary ratio of cracking stresses in shear and $45^{\circ}$ tension to be captured. The model is calibrated using the results of standard mechanical tests: $0^{\circ}$ tension, $45^{\circ}$ tension, and shear.

The predictive utility of the model has been demonstrated through comparisons of numerical simulation results with fullfield displacement and strain measurements in open-hole tension tests. The correlations are remarkably good. In particular, the model accurately predicts the size and shape of the shear bands that develop at the hole edge. Quantitative assessment of errors in model predictions have been made through comparisons of the displacement fields. The comparisons suggest that the progressively increasing errors at high stresses are attributable to non-proportional stressing within some material elements (notably, in the shear bands). The principal utility of the present model is expected to be in capturing CMC deformation in the intermediate stress regime: after elasticity ceases to apply but before effects of non-proportional stressing become important. Future work will focus on the effects of material softening and the onset of fracture in notched CMC panels.

\section{Acknowledgements}

This work was supported by the Pratt \& Whitney Center of Excellence at the University of California, Santa Barbara (monitored by Douglas Berczik), and the US AFOSR (Ali Sayir) and NASA (Anthony Calomino) under the National Hypersonics Science Center for Materials and Structures (AFOSR Prime Contract No. FA9550-09-1-0477 to Teledyne Scientific and Sub-contract No. B9U538772 to UCSB). VPR was supported in part by a National Defense Science and Engineering Graduate Fellowship. We 
acknowledge support from the Center for Scientific Computing at the CNSI and MRL: an NSF MRSEC (DMR-1121053) and NSF CNS0960316.

\section{Appendix A. Elastic constants of cross-ply composite}

The compliance matrix of a unidirectional ply (assumed to be in a state of plane stress) can be written in terms of the engineering constants as:

$\boldsymbol{S}_{0}=\left[\begin{array}{ccc}1 / E_{a} & -v_{t} / E_{t} & 0 \\ -v_{a} / E_{a} & 1 / E_{t} & 0 \\ 0 & 0 & 1 / G_{a}\end{array}\right]$

The engineering constants, in turn, are found using the generalized self-consistent scheme (GSCS) [30,31]. It is a micromechanical model that estimates the macroscopic elastic constants of the fiber composite using the elastic constants of the constituents. The GSCS model yields the same result as the cylindrical composites assemblage (CCA) model [42-44] for four of the five composite elastic constants:

$$
\begin{aligned}
& E_{a}=E_{m} V_{m}+E_{f} V_{f}+\frac{4\left(v_{f}-v_{m}\right)^{2} V_{m} V_{f}}{V_{m} / k_{f}+V_{f} / k_{m}+1 / G_{m}} \\
& v_{a}=v_{m} V_{m}+v_{f} V_{f}+\frac{\left(v_{f}-v_{m}\right)\left(1 / k_{m}-1 / k_{f}\right) V_{m} V_{f}}{V_{m} / k_{f}+V_{f} / k_{m}+1 / G_{m}} \\
& G_{a}=\frac{G_{m}\left(G_{m} V_{m}+G_{f}\left(1+V_{f}\right)\right)}{G_{m}\left(1+V_{f}\right)+G_{f} V_{m}} \\
& k=\frac{k_{m}\left(k_{f}+G_{m}\right) V_{m}+k_{f}\left(k_{m}+G_{m}\right) V_{f}}{\left(k_{f}+G_{m}\right) V_{m}+\left(k_{m}+G_{m}\right) V_{f}}
\end{aligned}
$$

where the subscripts $f$ and $m$ denote the fiber and effective medium, respectively. The shear modulus, $G$, and the transverse bulk modulus, $k$, are given by the expressions (for isotropic materials):

$$
\begin{aligned}
& G=\frac{E}{2(1+v)} \\
& k=\frac{G}{1-2 v}
\end{aligned}
$$

The remaining constant, the transverse shear modulus, $G_{t}$, is only bounded by the CCA model. An exact solution, however, can be found by using the GSCS formula given in [30] (the expression is quite lengthy and is thus not reproduced here). With these five constants, the transverse Young's modulus, $E_{t}$, and the transverse Poisson's ratio, $v_{t}$ can be computed using the formulae [43]:

$$
\begin{aligned}
v_{t} & =\frac{k-m G_{t}}{k+m G_{t}} \\
E_{t} & =2\left(1+v_{t}\right) G_{t}
\end{aligned}
$$

where

$m=1+\frac{4 k v_{a}^{2}}{E_{a}}$

The compliance matrix for the $90^{\circ}$ ply is obviously

$$
\boldsymbol{S}_{90}=\left[\begin{array}{ccc}
1 / E_{t} & -v_{a} / E_{a} & 0 \\
-v_{t} / E_{t} & 1 / E_{a} & 0 \\
0 & 0 & 1 / G_{a}
\end{array}\right]
$$

Then, using elasticity theory, the compliance matrix for the overall (cross-ply) composite is:

$\boldsymbol{S}^{\mathcal{C}}=2\left(\left[\boldsymbol{S}_{0}\right]^{-1}+\left[\boldsymbol{S}_{90}\right]^{-1}\right)^{-1}$

\section{References}

[1] Cady C, Mackin TJ, Evans AG. Silicon carbide/calcium aluminosilicate: a notchinsensitive ceramic-matrix composite. J Am Ceram Soc 1995;78(1):77-82.
[2] Genin GM, Hutchinson JW. Composite laminates in plane stress: constitutive modeling and stress redistribution due to matrix cracking. J Am Ceram Soc 1995;80(5):1245-55.

[3] Heredia FE, Spearing SM, Mackin TJ, He MY, Evans AG, Mosher P, et al. Notch effects in carbon matrix composites. J Am Ceram Soc 1994;77(11):2817-27.

[4] Levi CG, Yang JY, Dalgleish BJ, Zok FW, Evans AG. Processing and performance of an all-oxide ceramic composite. J Am Ceram Soc 1998;81(8):2077-86.

[5] Mackin TJ, Purcell TE, He MY, Evans AG. Notch sensitivity and stress redistribution in three ceramic-matrix composites. J Am Ceram Soc 1995;78(7):1719-28.

[6] Mackin TJ, Perry KE, Epstein JS, Cady C, Evans AG. Strain fields and damage around notches in ceramic-matrix composites. J Am Ceram Soc 1996;79(1):65-73.

[7] McNulty JC, Zok FW, Genin GM, Evans AG. Notch-sensitivity of fiber-reinforced ceramic-matrix composites: effects of inelastic straining and volumedependent strength. J Am Ceram Soc 1999;82(5):1217-28.

[8] Kramb VA, John R, Zawada LP. Notched fracture behavior of an oxide/oxide ceramic-matrix composite. J Am Ceram Soc 1999;82(11):3087-96.

[9] Mall S, Bullock DE, Pernot JJ. Tensile fracture behaviour of fibre-reinforced ceramic-matrix composite with hole. Composites 1994;25(3):237-42.

[10] Evans AG, Domergue JM, Vagaggini E. Methodology for relating the tensile constitutive behavior of ceramic-matrix composites to constituent properties. J Am Ceram Soc 1994;77(6):1425-35.

[11] Evans AG, Zok FW. The physics and mechanics of fibre-reinforced brittle matrix composites. J Mater Sci 1994;29(15):3857-96.

[12] Aveston J, Cooper GA, Kelly A. Single and multiple fracture. In: The properties of fiber composites. National Physical Laboratory, IPC Science and Technology Press Ltd.; 1971. p. 15-24.

[13] Budiansky B, Hutchinson JW, Evans AG. Matrix fracture in fiber-reinforced ceramics. J Mech Phys Solids 1986;34(2):167-89.

[14] Xia ZC, Carr RR, Hutchinson JW. Transverse cracking in fiber-reinforced, brittle matrix cross-ply composites. Acta Metall Mater 1993;41(8):2365-76.

[15] Xia ZC, Hutchinson JW. Matrix cracking of cross-ply ceramic composites. Acta Metall Mater 1994;42(6):1933-45.

[16] Cox BN, Zok FW. Advances in ceramic composites reinforced by continuous fibers. Curr Opin Solid State Mater Sci 1996;1(5):666-73.

[17] Talreja R. Continuum modelling of damage in ceramic matrix composites. Mech Mater 1991;12(2):165-80.

[18] Camus G. Modelling of the mechanical behavior and damage processes of fibrous ceramic matrix composites: application to a 2-D SiC/SiC. Int J Solids Struct 2000;37(6):919-42.

[19] Chaboche JL, Maire JF. New progress in micromechanics-based CDM models and their application to CMCs. Compos Sci Technol 2001;61(15):2239-46.

[20] Hahn HT. Nonlinear behavior of laminated composites. J Compos Mater 1973;7(2):257-71

[21] Jones RM. Analysis of nonlinear stress-strain behavior of fiber-reinforced composite materials. J Aircr 1977;15(12):1669-76.

[22] Sandhu RS. Nonlinear behavior of unidirectional and angle ply laminates. ] Aircr 1976;13(2):104-11.

[23] Rajan VP, Zok FW. Remediation of a constitutive model for ceramic composite laminates. Composites Part A 2013;52:80-8.

[24] Adams DF, Walrath DE. Current status of the Iosipescu test method. J Compos Mater 1987;21(6):494-507.

[25] Grédiac M, Pierron F, Vautrin A. The Iosipescu in-plane shear test applied to composites: a new approach based on displacement field processing. Compos Sci Technol 1994;51(3):409-17.

[26] Morton J, Ho H, Tsai MY, Farley GL. An evaluation of the Iosipescu specimen for composite materials shear property measurement. J Compos Mater 1992;26(5):708-50.

[27] Pierron F. Saint-Venant effects in the Iosipescu specimen. J Compos Mater 1998;32(22):1986-2015.

[28] Cady C, Heredia FE, Evans AG. In-plane mechanical properties of several ceramic-matrix composites. J Am Ceram Soc 1995;78(8):2065-78.

[29] Turner KR, Speck JS, Evans AG. Mechanisms of damage and failure in carbonmatrix composites subject to tensile and shear loading. J Am Ceram Soc 1995;78(7):1841-8.

[30] Christensen RM, Lo KH. Solutions for effective shear properties in three phase sphere and cylinder models. J Mech Phys Solids 1979;27(4):315-30.

[31] Christensen RM. A critical evaluation for a class of micro-mechanics models. ] Mech Phys Solids 1990;38(3):379-404.

[32] Yang QD, Rugg KL, Cox BN, Marshall DB. Evaluation of macroscopic and local strains in a three-dimensional woven $\mathrm{C} / \mathrm{SiC}$ composite. J Am Ceram Soc 2005:88(3):719-25.

[33] Flores S, Evans AG, Zok FW, Genet M, Cox B, Marshall D, et al. Treating matrix nonlinearity in the binary model formulation for 3D ceramic composite structures. Composites: Part A 2010;41(2):222-9.

[34] Aubard X. Mechanical behaviour models of 2-D SiC-SiC composites. Ph.D. thesis. University of Paris VI, France; 1992.

[35] Rajan VP, Zok FW. Matrix cracking of fiber-reinforced ceramic composites in shear. J Mech Phys Solids, Submitted for publication.

[36] Sloan SW, Abbo AJ, Sheng D. Refined explicit integration of elastoplastic models with automatic error control. Eng Comput 2001;18(1/2):121-54.

[37] Rajan VP, Rossol MN, Zok FW. Optimization of digital image correlation fo high-resolution strain mapping of ceramic composites. Exp Mech 2012;52(9):1407-21.

[38] He MY, Wu B, Suo Z. Notch-sensitivity and shear bands in brittle matrix composites. Acta Metall Mater 1994;42(9):3065-70. 
[39] Suo Z, Ho S, Gong X. Notch ductile-to-brittle transition due to localized inelastic band. J Eng Mater Technol 1993;115(3):319-26.

[40] Whitney JM, Nuismer RJ. Stress fracture criteria for laminate composites containing stress concentrations. J Compos Mater 1974;8(3):253-65.

[41] Awerbuch J, Madhukar MS. Notched strength of composite laminates: predictions and experiments-a review. J Reinf Plast Compos 1985;4(1):3-159.
[42] Hashin Z, Rosen BW. The elastic moduli of fiber-reinforced materials. J Appl Mech 1964;31(2):223-32.

[43] Hashin Z. Analysis of properties of fiber composites with anisotropic constituents. J Appl Mech 1979;46(3):543-50.

[44] Hashin Z. Analysis of composite materials-a survey. J Appl Mech 1983;50(3):481-505. 